\title{
Effects of Self-Criticism and Its Relationship with Depression across Cultures
}

\author{
Ayano Yamaguchi ${ }^{1} \&$ Min-Sun Kim ${ }^{2}$ \\ ${ }^{1}$ College of Foreign Studies, Reitaku University, Kashiwa, Chiba, Japan \\ ${ }^{2}$ Communicology, University of Hawaii at Manoa, Honolulu, USA \\ Correspondence: Ayano Yamaguchi, College of Foreign Studies, Reitaku University, 2-1-1 Hikarigaoka, \\ Kashiwa-shi, Chiba-ken 277-8686, Japan. Tel: 81-471-733-605. E-mail: ayamag13@reitaku-u.ac.jp
}

Received: November 18, 2012

Accepted: January 4, $2013 \quad$ Online Published: February 21, 2013

doi:10.5539/ijps.v5n1p1

URL: http://dx.doi.org/10.5539/ijps.v5n1p1

\begin{abstract}
The purpose of this study is to test the cross-cultural predictions of the associations between self-criticism and depression. The participants consisted of 642 undergraduates - 200 of them studying in Japan, and 442 of them studying in the United States (Las Vegas: 242; Hawaii: 200). The results indicated that independent self-construal in the U.S. and Japan is negatively associated with comparative self-criticism, which bolstered college students' taking criticism personally and, in turn, contributed to a high level of depression among participants. However, interdependent self-construal in Japan is positively associated with internalized self-criticism, which bolstered college students' taking criticism personally and, in turn, contributed to a high level of depression among participants. Discussion of these results and their implications is provided, followed by suggestions for future research on culture, self-criticism, the tendency to take criticism personally which leads to high level of depression, and the interventions and implications of such research.
\end{abstract}

Keywords: cross-cultural underpinning, self-criticism, depression, health, intervention

\section{Introduction}

Self-criticism has received increased attention as a marker for vulnerability to depression and other psychological disorders in adolescents and adults (Blatt, Hart, Quinlan, Leadbeater, \& Auerbach, 1993). This personality style is associated with fragile self-esteem and a fear of failure and scrutiny from others (Blatt et al., 1993). Self-criticism is hypothesized to develop in individuals with cold, rejecting, and controlling parents who make their love contingent on their child's achievements. This personality style is implicated in poor personal and social functioning and adjustment throughout adolescence and adulthood (Blatt, 2004). Researchers often discuss individuals' experiences with critical, rejecting parents who display inconsistent love (Thompson \& Zuroff, 2004). Children are likely to internalize these experiences to form negative internal working models of self, other, and self-other relationships. These experiences then have an impact on subsequent interpersonal interactions, thereby leading to a high level of depression (Blatt et al., 1993). However, this assertion has been recently called into question by cultural psychologists (Markus \& Kitayama, 1991, 1994), and by the cross-cultural investigation of self-criticism on depression. Specifically, the degree to which people engage in the two types of self-criticism patterns (comparative and internalized) can be a powerful impetus for action. The effect of these types of self-criticism on depression, however, has not been extensively examined in cross-cultural research.

The purpose of this study is to examine the cultural underpinnings of loss of self-criticism and the taking of criticism personally - or the absence of such loss. Drawing on an independence-interdependence theory of cultural self (Markus \& Kitayama, 1991, 1994), we assert that comparative self-criticism may not always be directly linked to depression in cultures that privilege independence. This is because such a perception can compromise the ever-important sense of the self as independent. In contrast, internalized self-criticism is likely to be unequivocally beneficial in cultures that privilege interdependence. To investigate this cross-cultural prediction of self-criticism, we examine depression. Furthermore, we test college students at three different locations.

This article is organized as follows. First, we review the differences between self-construal and depression as 
delineated in the psychological and communication literature. Second, we explicate two moderating factors - comparative self-criticism and internalized self-criticism - from a cross-cultural perspective, and propose a conceptual model specifying their respective influences on depression. Third, we describe a study conducted to test the conceptual model, followed by an analysis of the results. The concluding section discusses implications, limitations, and possible directions for future research.

\subsection{Self-Construal}

Markus and Kitayama $(1991,1994)$ found that Western cultures tend to construe the self as separate from its social context, and thus emphasize autonomy and independence, which is independent self-construal. In contrast, Eastern cultures conventionally construe and construct the self as a constituent of a broader social context; their concept of self entails characteristics and qualities of the social environment, which is interdependent self-construal. It is proposed that self-construal varies among cultures based on an emphasis on either independence or interdependence (Markus \& Kitayama, 1991, 1994). In middle-class Euro-American cultural contexts, which emphasize independence, the individuals engaged in these cultural views are motivated to discover and affirm positive dispositional attributes and then use them to guide and organize their thoughts and actions (Heine, Lehman, Markus, \& Kitayama, 1999). In contrast, in many Asian contexts, which emphasize interdependence, the individuals engaged in these cultural contexts are motivated to fit in and adjust themselves to expectations and the needs of others in a relationship (Morling, Kitayama, \& Miyamoto, 2002). Kitayama, Mesquita, and Karasawa (2006) demonstrated that Japanese people were more likely to experience socially engaging emotions (which are linked to one's interdependence, e.g., friendly feelings and guilt) than socially disengaging emotions (which are linked to one's independence, e.g., pride and anger). In contrast, Americans were more likely to experience socially disengaging emotions than socially engaging emotions (Mesquita \& Karasawa, 2002). Another important factor in the pursuit of positivity of self is the self-serving attribution of success and failure. Researchers have identified that Euro-Americans take credit for their successes and blame others or contextual factors for their failures. Such a biased attribution notion and pattern is not always common among Japanese, who are more balanced in their assessment of success and failure. Japanese are more likely to focus on their shortcoming (Kitayama, Takagi, \& Matsumoto, 1995).

\subsection{Self-Criticism}

The Levels of Self-Criticism (LOSC) Scale was developed as a two-dimensional self-criticism concept by Thompson and Zuroff (2004). This scale measures two developmental levels of self-criticism: Comparative Self-Criticism (CSC) and Internalized Self-Criticism (ISC). First, CSC is defined as a negative perspective and view of the self as compared to others. The focus is on the unfavorable comparison of the self with others, who are seen as superior and hostile, or critical. Thus, one of the characteristics of CSC is interpersonal hostility (Thompson \& Zuroff, 2004). In the context of this unfavorable comparison of the self with others, there is discomfort at the prospect of being evaluated or exposed to others, which may be linked to the independent self-construal seen in Western contexts. That is, rather than viewing one's shortcomings in comparison to ideals formulated in one's own mind, Westerners are more likely to view their own shortcomings in comparison to others. They may do this through CSC, self-enhancement, and independence, seeking to confirm their positive internal attributes of self and self-esteem. Secondly, ISC is defined as a negative perspective and view of the self as compared to internal, personal standards. ISC does not involve comparing oneself with others, but viewing oneself as deficient. The issue here is a negative response to previously defined success (Thompson \& Zuroff, 2004), and responding to success by seeing it as failure. This may be linked to interdependent self-construal in cross-cultural contexts. That is, rather than viewing one's own shortcomings in comparison to others, Japanese people are more likely to show ISC.

\subsection{Cultural Variations in Self-Criticism and Depression}

Heine et al. (1999) recognized that self-criticism plays a key role for East Asians in their endeavor to better themselves and maintain good face. Face can be understood as a communal property that is loaned from societies to individuals who occupy particular positions in social networks (Mao, 1994). In cross-cultural contexts, East Asians are likely to use a number of psychological processes - including the positive and functional use of self-critical tendencies - to gain and maintain face, in the same way that Europeans and Americans use various psychological processes to gain and maintain self-esteem. In simple terms, "taking criticism personally" may be considered one of the mechanisms used to keep face and maintain functionality. Cultural differences in self-construal may play a significant role in the experience and reporting of depression and mental health disorders caused by social behaviors. In their theoretical model of well-being, Kitayama and Markus (2000) propose that in Western cultural contexts, well-being results from the pursuit of happiness and life according to 
middle-class American values and practices in the United States, which include "comparative self-criticism." In contrast, they also suggest that when their model of well-being is applied in non-Western cultural contexts, for example in Japan, a higher level of self-critical tendencies would be required - including "internalized self-criticism," failure, and suffering rather than personal feelings of happiness. These are necessary experiences in non-Western cultures that should be overcome before one can find the path to "being well." The principal values in this context are associated with a culture of self-criticism, self-improvement, and mutually sympathetic relationships, which might be related to self-control, functionality, effort, social roles, filial piety, community values, warm-heartedness, empathy, and taking perspective. In the Japanese cultural context, initial evidence suggests that this attitude and orientation are often necessary for personal achievement because they may lead to the recognition of one's own shortcomings and, therefore, the initiative to work hard (Heine et al., 1999; Kitayama, Markus, \& Kurosawa, 2000). Furthermore, a self-critical attitude and orientation enable the individual to fully participate in mutually sympathetic social relations (Kitayama \& Markus, 2000).

\subsection{Proposed Model: Integrative Framework}

The purpose of the present study is to test the above-mentioned cross-cultural predictions of the associations between self-criticism and depression. On one hand, among Euro-Americans, the relationship between perceived independent self-construal and depression would be strong and negative; even when such a relationship existed, it would be entirely moderated by CSC. On the other hand, among Asians, the relationship between perceived interdependent self-construal and depression would be strong and positive. Moreover, this relationship would not be moderated by ISC. Americans more readily endorse positive self-characteristics and prefer more dominant and more direct (as opposed to avoidance) communication strategies (Kim, 2002). When considering the mechanisms and patterns associated with self-criticism in the United States, it seems that CSC rather than ISC is more likely to play a role in depression. The Japanese, unlike people in the United States, more readily endorse negative self-characteristics (Heine \& Lehman, 1995, 1999) and prefer avoidance-type, as opposed to dominant-type, communication strategies (Kim, 2002). Therefore, strategies and behaviors that seem unfavorable to those in the United States may be socially advantageous to the Japanese (Kim, 2002). When considering the mechanisms and patterns of self-criticism seen among the Japanese, ISC rather than CSC is more likely to be linked to depression. To elaborate, a Japanese person is more likely to be concerned about his or her own shortcomings as compared to his or her own internal standards (ISC), rather than as compared to others (CSC).

Exploring the effects of self-construal on depression, comparative/internalized self-criticism, and the tendency to take criticism personally is difficult yet certainly necessary because this research area has been neglected in the psychological and communication literature. To date, few studies have attempted to link severe levels of depression with the individual's level of comparative/internalized self-criticism, and taking criticism personally. A few studies have attempted to link levels of self-construal with depression, but self-construal has not been associated with comparative and internalized self-criticism in relation to a high level of depression. Finally, none of the studies have been linked together in order to explain why people systematically the tendency to take criticism personally, which, in turn, can lead to a high level of depression.

The main purpose of this study is to propose and test a model that delineates the process through which dimensions of self-construal influence depression. The model also incorporates anti-coping behaviors (comparative and internalized self-criticism, taking criticism personally) as potential contributors to depression. Based on cultural variations related to self-criticism and taking criticism personally, we hypothesize different relationships as predictors of depression across different cultures. This is tested in three cultural samples - the U.S. mainland, Hawaii, and Japan - using the modified model we propose, which is based on Kitayama and Markus's cultural validation of subjective well-being model (2000). Attention is specifically focused on the internal structure of self-construal as expressed across three different locations. A graphical presentation of the conceptual model linking these factors is illustrated in Figure 1. 


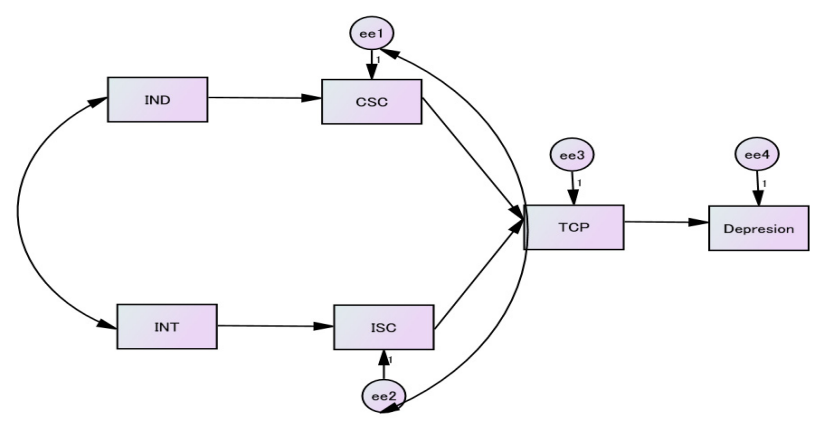

Figure 1. Proposed cross-cultural conceptual self-criticism in depression model

*CSC: Comparative Self-Criticism, ISC: Internalized Self-Criticism, TCP: Taking Criticism Personally

\section{Method}

\subsection{Participants and Procedures}

The participants in this study consisted of 242 undergraduate students enrolled at a university in Las Vegas (150 males, 92 females, mean age $=21$ years, and $\mathrm{SD}=3.57$ ); 200 undergraduate students enrolled at a university in Hawaii (108 males, 92 females, mean age $=20$ years, and $\mathrm{SD}=2.30$ ); and 200 mostly undergraduate students enrolled at a university in Kashiwa City, Japan (74 males, 126 females, mean age $=19$ years, and SD $=1.51$ ). The researchers invited students who were taking several courses at their respective universities to participate in this study. Volunteers, who were recruited in compliance with ethical procedures for human subjects, were permitted to withdraw from the study at any time, and were assured anonymity. The participants completed a survey concerning measures of self-criticism, "taking criticism personally," and depression. The wording of the questionnaires developed for the Japanese sample was translated and back-translated by a bilingual and bicultural professional to ensure cross-cultural equivalence in meaning (Brislin, 1970). Once the participants had completed the questionnaire, they returned it to the researchers.

\subsection{Analysis Plan}

To establish measurement invariance, all scales were subjected to Confirmatory Factor Analysis (CFA) using AMOS 18.0 (Arbuckle, 2006), which was employed along with a maximum likelihood estimation. To assess the scales in this study, a CFA was performed, and its outcome indicated that this measurement model was an acceptable fit except for the chi-square test. However, the chi-square test, by itself, is not necessarily an indicator of a poor fit (Bagozzi \& Yi, 1988). A non-significant goodness-of-fit $\chi^{2}$ value is desired, but this may be unlikely with large samples (Kline, 1998). To overcome this weakness, many researchers use an alternative fit index, such as the root mean square error of approximation (RMSEA) and the goodness of fit index (GFI), neither of which is influenced by sample size. The GFI value of an alternative index, which indicates absolute or relative proportions of the observed covariance if it is above .90, is usually considered satisfactory (Kline, 1998). RMSEA values between 0.1 and 0.08 represent a mediocre fit; values between 0.08 and 0.05 indicate a reasonable fit; and those under 0.05 signify a fit that is close to approximating the population (Byrne, 2001). In the path model, both moderators-comparative and internalized self-criticisms and taking criticism personally - were included in the analysis, thus providing simultaneous tests. Path analysis, a type of Structural Equation Modeling (SEM) using AMOS 18.0, was performed to test the moderating role of self-criticisms on self-construal and depression. In the tested model, only paths with a significance level of .05 or less, based on the standard cutoff $t$ value of 1.96, were retained for model parsing (Hatcher, 1994).

\subsection{Measures}

The measures for Independent self-construal (IND) and Interdependent self-construal (INT) were taken using self-construal scales (Leung \& Kim, 1997). Responses to the 29 items were measured on a 7-point scale, ranging from 1 (strongly disagree) to 7 (strongly agree). Higher scores indicated a higher level of independent and interdependent self-construal. The measurement invariance models fit the data well: RMSEA $=.041, \mathrm{GFI}=.980$, (IND) and RMSEA $=.096$, GFI $=.940$ (INT). Cronbach's alphas were .75 (IND) and .75 (INT). Thus, the first 
measurement models were accepted because other indices had good values in this study.

Self-criticism was measured using the LOSC scale (Thompson \& Zuroff, 2004). Responses to 22 items were measured on a 7-point scale, ranging from 1 (strongly disagree) to 7 (strongly agree). Higher scores indicated a higher level of self-criticism. The measurement invariance models fit the data well: RMSEA $=.042, \mathrm{GFI}=.995$ $(\mathrm{CSC}), \mathrm{RMSEA}=.058, \mathrm{GFI}=.972$ (ISC). Cronbach's alphas were $.70(\mathrm{CSC})$ and .82 (ISC). Thus, the first measurement models were accepted because other indices had good values in this study.

Taking criticism personally was measured with the Bothered by Criticism Scale (Fitzpatrick, 1996). This is a measure of personal reactions to criticism from others. The scale consists of 18 items asking respondents to rate how often they experience these reactions when they encounter criticism. Ratings were assigned on a 7-point scale, ranging from 1 (strongly disagree) to 7 (strongly agree). Higher scores indicated a higher level of "taking criticism personally." The measurement invariance models fit the data well: RMSEA $=.037$, GFI $=.992$. Cronbach's alpha was .90 . Thus, the first measurement models were accepted because other indices had good values in this study.

Symptoms of Depression was measured using the Center for Epidemiological Studies Depression Scale (CES-D) (Radloff, 1977). It consists of 20 items in which respondents are asked to rate how often they have had particular depressive thoughts or feelings during the past week on a 7-point scale, ranging from 1 (none of the time) to 7 (all of the time). Higher scores indicated a higher level of depression. The measurement invariance models fit the data well: RMSEA $=.076, \mathrm{GFI}=.983$; Cronbach's alpha was .91 . Thus, the first measurement models were accepted because other indices had good values in this study.

\section{Results}

\subsection{Preliminary Analyses}

Descriptive statistics for the study variables are reported in Table 1. Table 1 shows the mean and standard deviations for all study variables. A correlation analysis was conducted to assess the potential for multicollinearity in the data analysis $(\mathrm{p}<0.05$, see Table 2$)$.

Table 1. Means, standard deviations for study measures in Japan, Hawaii, and the U.S. mainland

\begin{tabular}{lllllll}
\hline & Japanese & & Hawaii & \multicolumn{2}{c}{ U.S. Mainland } \\
& $\mathrm{M}$ & $\mathrm{SD}$ & $\mathrm{M}$ & $\mathrm{SD}$ & $\mathrm{M}$ & $\mathrm{SD}$ \\
\hline 1.ID & 5.23 & 1.04 & 5.9 & 0.68 & 5.86 & 0.93 \\
2.INT & 4.73 & 1.18 & 5 & 0.95 & 4.7 & 1.03 \\
3.CSC & 4.03 & 0.87 & 2.84 & 0.77 & 3 & 1.02 \\
4.ISC & 4.81 & 1.05 & 5.20 & 1.23 & 5 & 1.3 \\
5.TCP & 4.64 & 1.26 & 3.30 & 1.41 & 3.06 & .1 .50 \\
6.Depression & 1.12 & 0.72 & 3.30 & 1.15 & 1.32 & -1.26 \\
\hline
\end{tabular}

Note. IND = Independent Self-Construal; INT = Interdependent Self-Construal; CSC = Comparative Self-Criticism; ISC = Internalized Self-Criticism; TCP = Taking Criticism Personally

Table 2. Correlation of variables in the multivariate model

\begin{tabular}{|c|c|c|c|c|c|c|}
\hline & 1 & 2 & 3 & 4 & 5 & 6 \\
\hline 1. ID & - & $.158^{* *}$ & $-466 * *$ & $.165^{* *}$ & $-.254^{* *}$ & -.043 \\
\hline 2.INT & & - & -0.077 & $.132 * *$ & $.156^{* *}$ & $.141 * *$ \\
\hline 3. CSC & & & - & $-.109 * *$ & $.448^{* *}$ & 0.031 \\
\hline 4.ISC & & & & - & $.147^{* *}$ & $.209^{*}: *$ \\
\hline 5.TCP & & & & & - & $.197 * *$ \\
\hline 6.Depression & & & & & & - \\
\hline
\end{tabular}

Note. IND $=$ Independent Self-Construal; INT $=$ Interdependent Self-Construal; $\mathrm{CSC}=$ Comparative

Self-Criticism; ISC = Internalized Self-Criticism; TCP = Taking Criticism Personally. The number in parentheses 
indicates the number of scale items

\subsection{Test of the Path Model}

We assumed that perceived comparative self-criticism is not always directly linked to depression in cultures that privilege independence because this perception can compromise the ever-important sense of the self as independent. In contrast, perceived internalized self-criticism is likely to be unequivocally lacking in cultures that privilege interdependence. In order to identify the cross-cultural prediction of self-criticisms in depression, we attempted to examine depression. We also assumed that the perception of taking criticism personally is directly positively linked to depression in cultures that privilege interdependence, rather than independence. Furthermore, in order to verify this, we tested college students at three different locations.

We conducted formal path analyses involving four sets of variables (two individual-level dimensions of self-construal, two levels of self-criticism, one level of taking criticism personally, and one level of depression). Specifically, it was predicted that the greater the individual's construal of self as independent, the greater the individual levels of self-criticism and taking criticism personally, which, in turn, would lead to a higher level of depression. It was also predicted that the greater the individual's construal of self as interdependent, the more self-critical and the greater the tendency to take criticism personally, which, in turn, would lead to a higher level of depression. The predictors were tested by analyzing the proposed theoretical path model. The analysis corrects for underestimation of coefficients, and thus, it provided us with an accurate picture of the relationships among the variables.

Our major research objective was to propose and test a model that delineates the process by which dimensions of self-construals influence depression. The model also incorporates anti-coping resources (comparative and internalized self-criticism; taking criticism personally) as potential contributors to depression. One path analysis was conducted to test the integrated model as it relates to the effects of self-construals, self-criticism, and taking criticism personally on depression. The fit of the model presented in Figure 2-4 was good, except for the chi-square test, $\chi^{2}(24)=94.55, \mathrm{p}<.001$ (RMSEA $\left.=.06, \mathrm{GFI}=.96\right)$. As shown in Figure 2-4, all coefficients are significant $(\mathrm{p}>.05)$ and in the proposed direction. As shown in Figures 2 and 3, for participants in the United States, independent self-construal negatively influence perceived comparative self-criticism $(\beta=-.42, t=-7.35$, $p<.01 ; \beta=-.37, t=-5.58, p<.01)$. Perceived comparative self-criticism positively influences taking criticism personally $(\beta=.36, t=5.95, p<.05 ; \beta=.38, t=5.84, p<.01)$. Taking criticism personally positively influences depression $(\beta=.31, t=5.10, p<.01 ; \beta=.45, t=7.12, p<.01)$. Interdependent self-construals do not significantly influence perceived internalized self-criticism $(\beta=.09, t=1.46, p>.05 ; \beta=.12, t=1.65, p>.05)$. Perceived internalized self-criticism positively influences taking criticism personally $(\beta=.20, t=3.33, p<.01 ; \beta$ $=.17, t=2.64, p<.01$ ). As shown in Figure 4, for participants in Japan, independent self-construals negatively influence perceived comparative self-criticism $(\beta=-.32, t=-4.87, p<.01)$. Perceived comparative self-criticism positively influences taking criticism personally $(\beta=.13, t=2.06, p<.05$ ). Taking criticism personally positively influences depression $(\beta=.31, t=4.65, p<.01)$. Interdependent self-construals positively influence perceived internalized self-criticism $(\beta=.18, t=2.65, p<.01)$. Perceived internalized self-criticism positively influences taking criticism personally $(\beta=.40, t=6.25, p<.01)$.

Overall, these results demonstrate that all hypothesized models are supported. As expected, independent self-construals in all three locations are negatively associated with comparative self-criticism, which bolstered college students' ratings for taking criticism personally, and in turn, contributed to a high level of depression among participants; however, interdependent self-construals in Japan are positively associated with internalized self-criticism, which bolstered college students' ratings for taking criticism personally, and in turn, contributed to a high level of depression among participants. 


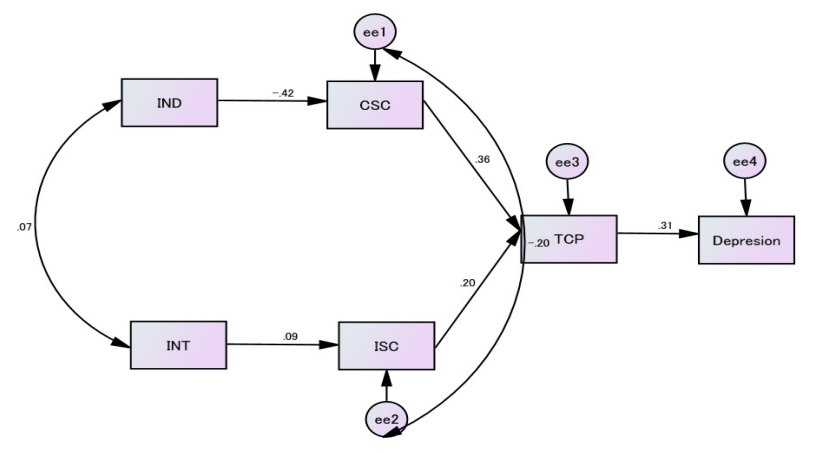

Figure 2. Final model of self-criticism and depression on the U.S. mainland

*CSC: Comparative Self-Criticism, ISC: Internalized Self-Criticism, TCP: Taking Criticism Personally

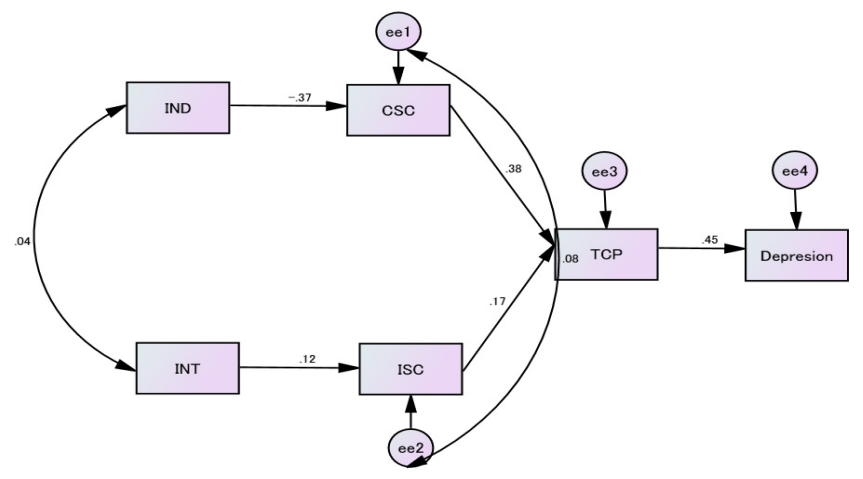

Figure 3. Final model of self-criticism and depression in Hawaii

*CSC: Comparative Self-Criticism, ISC: Internalized Self-Criticism, TCP: Taking Criticism Personally

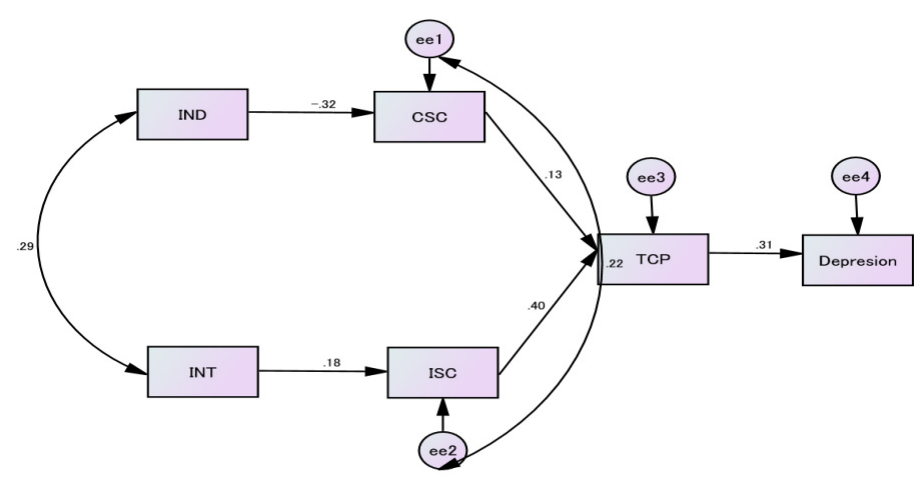

Figure 4. Final model of self-criticism and depression in Japan

*CSC: Comparative Self-Criticism, ISC: Internalized Self-Criticism, TCP: Taking Criticism Personally 


\section{Discussion and Conclusion}

The purpose of this study was to explore the relationships between self-construals, self-criticism, and depression. The results indicated that independent self-construal in all three locations (Japan, Hawaii, and the U.S. mainland) was negatively associated with comparative self-criticism, which raised college students' tendency to take criticism personally and, in turn, contributed to higher levels of depression among participants. However, interdependent self-construal in Japan was positively associated with internalized self-criticism, which raised these college students' tendencies to take criticism personally and, in turn, contributed to higher levels of depression among Japanese participants. Our study also indicated that independent self-construal, comparative self-criticism, and taking criticism personally in all three locations were better predictors of depression than interdependent self-construal.

One main finding was that with regards to depression in the three locations, there was no cross-cultural difference in the role of independent self-construal or in that of comparative self-criticism (comparing oneself to others to identify one's own shortcomings). Beyond cultural differences in depression, it was found that independent self-construal played a significant role in the relationship between comparative self-criticism, taking criticism personally, and depression. People who have higher independent self-construals are more likely to emphasize their uniqueness and originality. Within an interpersonal communication context, if people have higher independent self-construals, they are more likely to engage in comparative self-criticism and to take criticism personally, leading to higher levels of depression. We found that interdependent self-construal in Japan was also a better predictor of depression than independent self-construal. The findings from this study are in line with the traditional Japanese belief that the individual's interdependent self-construal, internalized self-criticism, and "taking criticism personally" seem to be derived from, and are conferred to, the individual by people who are related to the self. Thus, Japanese people are more likely to focus on their own shortcomings. The question then arises as to whether interdependent self-construal is more useful than independent self-construal in predicting internalized self-criticism and taking criticism personally. For the Japanese sample, we cautiously speculate that because self-criticism and taking criticism personally are primarily means of maintaining face, interdependent self-construal may become a more salient variable in predicting higher levels of depression.

Another plausible explanation as to why the top portion of the model was not consistent with the data could be simply that there is another psychological process than self-criticism or taking criticism personally involved for people with independent self-construals. For example, as stated above, apology was chosen most, regardless of which self-construal the person emphasized. Because self-criticism and taking criticism personally are ultimately forms of face work (Goffman, 1971), it might be interesting for future researchers to replace self-criticism or taking criticism personally with self- vs. other-face maintenance.

In conclusion, we have shown that the effect of perceived self-criticisms on depression is moderated by culture. In Euro-American independent cultural contexts, the link between independent self-construal and comparative self-criticism (which leads to explicit self-criticism) and depression did exist among college students in all three locations; it was also entirely moderated by comparative self-criticism. In contrast, only for the Japanese was the link between perceived interdependent self-construal and internalized self-criticism and depression more direct. This is consistent with the hypothesis that perceived internalized self-criticism, which may be provided by implicit self-criticism, affirms the ever-important sense of the self as interdependent and connected with others. Importantly, the current work provides the first evidence in the research literature that cultural variation exists among college students in all three locations. Moreover, it applies to measures of both subjective well-being and physical health. We showed that the effectiveness of perceived self-criticism in enhancing subjective well-being and health is indeterminate until the nature of the cultural context is fully taken into account.

\section{Implications}

The findings of this study partially support, develop, and expand our proposed modified theory based on cultural variations in subjective well-being theory (Kitayama \& Markus, 2000). Using the theory of cultural validation of subjective well-being, this study develops an integrative theoretical framework to identify the effect of cultural validation and cultural underpinnings on depression. This study suggests alternative theoretical explanations and interpretations. According to the findings, Asian people are more likely to emphasize internalized self-criticism and to take criticism personally, resulting in high levels of depression. This study found that this alternative explanation and interpretation are likely to be implausible, because in Asian contexts the effect of internalized self-criticism on depression is more likely to stem from their shortcomings. This leads to a relational orientation from the perspective of cultural affordance in depression that is consistent with a person's shortcomings, negative states, and misfortune (Kitayama \& Markus, 2000; Kitayama, \& Uchida, 2005; Uchida, Kitayama, 
Mesquita, Reyes \& Morling, 2008). Further research is needed to explore when perceived internalized self-criticism, which leads to relational orientation, may have positive effects on depression in Asians. Further research is also needed to explore when perceived comparative self-criticism, which leads to a sense of efficacy and control from the viewpoint of cultural affordance, may have negative effects on depression in Euro-Americans.

\section{Acknowledgements}

This research was partly supported by Grant-in-Aid for Young Scientists B, Japan Science Technology (JST) Agency, Japan. The authors thank Dr. Atsushi Oshio for his invaluable assistance in the statistical analysis for this research study. We also express gratitude to Dr. Arlie Tagayuna, Hyun Hee Heo, Krista Hodges, Jayne Bopp, Prof. Emeritus Hideyuki Kudo, Prof Emeritus Kyoko Yashiro, Prof. Shujiro Mizuno, and Prof. Satomi Kurosu for their assistance in collecting the study data and for their helpful advice on earlier versions of this manuscript.

\section{References}

Arbuckle, J. L. (2006). AMOS (Version 7.0) [computer program]. Chicago: SPSS.

Bagozzi, R. P., \& Youjae, Yi. (1988). On the Evaluation of Structural Equation Models. Journal of the Academy of Marketing Science, 16(Spring), 74-94. http://dx.doi.org/10.1007/BF02723327

Blatt, S. J. (2004). Experiences of depression: Theoretical, clinical and research perspectives. Washington, DC: American Psychological Association. http://dx.doi.org/10.1037/10749-000

Blatt, S. J., Hart, B., Quinlan, D. M., Leadbeater, B., \& Auerbach, J. (1993). Interpersonal and self-critical dysphoria and behavioral problems in adolescence. Journal of Youth and Adolescence, 22, 253-269. http://dx.doi.org/10.1007/BF01537791

Brislin, R. W. (1970). Back-translation for cross-cultural research. Journal of Cross-Cultural Psychology, 1(3), 185-216. http://dx.doi.org/10.1177/135910457000100301

Byrne, B. M. (2001). Structural Equation Modeling with AMOS: Basic Concepts, Applications, and Programming. Mahway, NJ: Lawrence Erlbaum Associates.

Fitzpatrick, D. K. (1996). Sensitivity to criticism and vulnerability to depression. Proquest Dissertations and Theses. Canada: University of Waterloo (Canada).

Goffman E. (1971). Relations in Public: Microstudies of the Public Order. Basic Books: New York.

Hatcher, L. (1994). A step-by-step approach to using the SAS system for factor analysis and structural equation modeling. Cary, NC: SAS Institute.

Heine, S. J., \& Lehman, D. R. (1995). Cultural variation in unrealistic optimism: Does the West feel more invulnerable than the East? Journal of Personality \& Social Psychology, 81, 599-615. http://dx.doi.org/10.1037/0022-3514.81.4.599

Heine, S. J., \& Lehman, D. R. (1999). Culture, self-discrepancies, and self-satisfaction. Personality \& Social Psychology Bulletin, 25, 915-925. http://dx.doi.org/10.1177/01461672992511001

Heine, S. J., Lehman, D. R., Markus, H. R., \& Kitayama, S. (1999). Is there a universal need for positive self-regard? Psychological Review, 106, 766-794. http://dx.doi.org/10.1037/0033-295X.106.4.766

Kim, M. S. (2002). Non-western perspectives on human communication: Implications for theory and practice. Thousand Oaks, CA: Sage.

Kim, M. S., Wilson, S. R., Anastasiou, L., Aleman, C., Oetzel, J., \& Lee, H. R. (2009). The relationship between self-construals and perceived face threats in influence goals. Journal of International and Intercultural Communication, 2, 318-343. http://dx.doi.org/10.1080/17513050903177326

Kitayama S., Markus H. R., \& Kurosawa, M. (2000). Culture, emotion, and well-being: good Feelings in Japan and the United States. Cognition \& Emotion, 14, 93-124. http://dx.doi.org/10.1080/026999300379003

Kitayama, S., \& Markus, H. R. (2000). The Pursuit of Happiness and the Realization of Sympathy: Cultural Patterns of Self, Social Relations, and Well-Being. In E. D. Diener, \& E. M. Suh (Eds.), Culture and subjective well-Being (pp. 113-161). Cambridge, MA: MIT Press.

Kitayama, S., Mesquita, B., \& Karasawa, M. (2006). Cultural affordances and emotional experience: Socially engaging and disengaging emotions in Japan and the United States. Journal of Personality and Social Psychology, 91, 890-903. http://dx.doi.org/10.1037/0022-3514.91.5.890 
Kitayama, S., Takagi, H., \& Matsumoto, H. (1995). Causal attribution of success and failure: Cultural psychology of the Japanese self. Japanese Psychological Review, 38, 247-280.

Kitayama, S., \& Uchida, Y. (2005). I nterdependent Agency: An Alternative System for Action.

Kline, R. (1998). Principles and practice of structural equation modeling. New York, NY: The Guilford Press.

Leung, T., \& Kim, M. S. (1997). A Revised Self-Construal scale. Department of Speech, University of Hawaii at Manoa, Honolulu, Hawaii.

Mao, L. R. (1994). Beyond politeness theory: Face revised and renewed. Journal of Pragmatics, 21, 451-486. http://dx.doi.org/10.1016/0378-2166(94)90025-6

Markus, H. R., \& Kitayama, S. (1991). Culture and the self: Implications for cognition, emotion, and motivation. Psychological Review, 98, 224-253. http://dx.doi.org/10.1037/0033-295X.98.2.224

Markus, H. R., \& Kitayama, S. (1994). A collective fear of the collective: Implications for selves and theories of $\begin{array}{llll}\text { selves. Personality and Social Psychology Bulletin, 20, 568-579. } & \text {. }\end{array}$ http://dx.doi.org/10.1177/0146167294205013

Mesquita, B., \& Karasawa, M. (2002). Different emotional lives. Cognition and Emotion, 16, 127-141. http://dx.doi.org/10.1080/0269993014000176

Morling, B., Kitayama, S., \& Miyamoto, Y. (2002). Cultural practices emphasize influence in the United States and adjustment in Japan. Personality and Social Psychology Bulletin, 28, 311-323. http://dx.doi.org/10.1177/0146167202286003

Radloff, L. (1977). The CES-D scale: A self report depression scale for research in the general population. Applied Psychology Measurement, 1, 385-401. http://dx.doi.org/10.1177/014662167700100306

Thompson, R., \& Zuroff, D. C. (2004). The levels of self-criticism scale: comparative-self-criticism and internalized self-criticism. Personality \& Individual Differences, $36,419-430$. http://dx.doi.org/10.1016/S0191-8869(03)00106-5

Uchida, Y., Kitayama, S., Mesquita, B., Reyes, J. A. S., \& Morling, B (2008). Is Perceived emotional support beneficial? Well-being and health in independent and interdependent cultures. Personality and Social Psychology Bulletin, 34, 741-754. http://dx.doi.org/10.1177/0146167208315157 